TRANSACTIONS OF THE

AMERICAN MATHEMATICAL SOCIETY

Volume 276, Number 1, March 1983

\title{
RIGIDITY OF PSEUDOCONFORMAL CONNECTIONS
}

\author{
BY
}

MICHAEL MARKOWITZ AND ROGER SCHLAFLY

\begin{abstract}
Let $M^{2 n-1}(n \geqslant 3)$ be a strictly pseudoconvex abstract CR-hypersurface $\mathrm{CR}$-immersed in the unit sphere in $\mathrm{C}^{N}$. We show that the pseudoconformal connection induced on $M$ by the standard flat connection agrees with the intrinsic normal connection of Cartan-Chern-Tanaka if and only if $M$ is pseudoconformally flat. In this case $M$ is a piece of the transverse intersection of $S^{2 N-1}$ with a complex $n$-plane in $\mathbf{C}^{N}$.
\end{abstract}

1. Introduction. Let $M$ be an abstract strictly pseudoconvex CR-hypersurface of dimension $2 n-1, n \geqslant 3$, pseudoconformally immersed in the unit sphere $S^{2 N-1}$ in $C^{N}$. There are then two natural pseudoconformal connections defined on $M$ : the intrinsic (normal) connection of Cartan-Chern-Tanaka [2] and the connection induced on $M$ by the standard flat pseudoconformal connection on $S^{2 N-1}$ [3].

The purpose of this note is to sketch a proof of the following rather surprising result.

THEOREM. The induced pseudoconformal connection on $M$ is normal if and only if $M$ is auto-parallel in $S^{2 N-1}$ in the CR-sense. In this case $M$ is pseudoconformally flat and lies in $S^{2 N-1}$ as the transverse intersection of $S^{2 N-1}$ with a complex n-plane in $\mathbf{C}^{N}$.

For a hypersurface $M^{2 n-1}$ in $S^{2 n+1}$ this had been observed by S. Kobayashi in 1976.

Now suppose that $f$ is a proper holomorphic mapping from the unit ball $B^{n}$ in $\mathbf{C}^{n}$, $n \geqslant 3$, to the unit ball in $\mathbf{C}^{N}$. If $f$ extends holomorphically to a neighborhood of $S^{2 n-1}$, then $M$, the image of $S^{2 n-1}$ under $f$, is a pseudoconformally immersed submanifold of $S^{2 N-1}[6$, p. 313]. The theorem then implies the following

COROLlary. If the induced pseudoconformal connection on $M$ is normal, then the image of $f$ lies in a complex n-plane in $\mathrm{C}^{N}$.

Alexander's theorem [6, p. 316] then implies that, up to composition on the right and left with an automorphism, $f$ is simply the restriction to $B^{n}$ of the natural inclusion of $\mathbf{C}^{n}$ into $\mathbf{C}^{N}$.

In codimension one the hypothesis in the corollary is automatically satisfied. In this case the conclusion is due to $\mathrm{S}$. Webster [8], who proved it under the assumption that $f$ extends to a $C^{3}$ immersion of $S^{2 n-1}$ into $S^{2 N-1}$. We expect that in the general

Received by the editors November 23, 1981.

1980 Mathematics Subject Classification. Primary 32F25; Secondary 53B15, 53B25.

(c) 1983 American Mathematical Society 0002-9947/81/0000-0103/\$01.75 
case also this result holds under considerably weaker assumptions on the boundary regularity of $f$.

REMARK. The Cartan-Janet theorem in Riemannian geometry may be interpreted as the assertion that the Levi-Civita connection on a real analytic Riemannian manifold may be realized locally, via a real analytic imbedding, as the connection induced on a submanifold of $\mathbf{R}^{n}$ by the flat Euclidean connection. The projective analogue of this result is due to $S$. S. Chern [1].

Chern showed that a torsionfree projective connection whose curvature satisfies a certain Bianchi condition may be realized locally as the connection induced on a submanifold of projective space by the standard flat projective connection and an appropriate choice of normal framing. (Again this is all in the real analytic category as the proof relies on the Cartan-Kähler theorem.) Recently one of us has proven the analogue of Chern's result in conformal geometry [4].

This evidence naturally leads one to conjecture that such an imbedding result holds in every geometry modeled on a flat semisimple homogeneous space (see [5]), at least in those cases in which the underlying graded Lie algebra is of classical type.

The above theorem however shows that the corresponding imbedding problem in pseudoconformal geometry does not in general admit a solution. In other words, among the normal pseudoconformal connections only the flat one may be realized as the connection induced on a submanifold of the flat model space by the standard flat connection. It would be interesting to know if such problems arise in geometries modeled on other "generalized" semisimple graded Lie algebras [7].

2. Proof of the Theorem. For definitions and most of the details in the following argument, the reader is referred to [3].

That the induced connection on an auto-parallel submanifold of $S^{2 N-1}$ is flat, hence normal, follows immediately from the pseudoconformal analogue of the Gauss equation (see [3, (6.8)]).

For the converse, let $R_{J}{ }_{\rho \bar{\sigma}}$ denote the curvature components of the induced connection with respect to an admissible coframe field adapted to $M$. Then according to [3, (6.4)] we have $R_{i k \bar{l}}{ }^{j}=-H_{i}{ }^{P} H_{P}{ }^{j} \bar{l}$, where $H_{i j}{ }^{P}$ denotes the "essential" components of the second fundamental form of the immersion (see [3, (4.11)]). Here we have used the Levi form to raise and lower indices as usual.

Now if the induced connection is normal, then in particular $R_{i k}^{j}{ }^{k}=0$; consequently $H_{i}{ }_{k}{ }_{k} H^{j}{ }_{P}{ }^{k}=0$. Contracting the indices $i$ and $j$ then yields $\left\|H_{i}{ }_{j}\right\|=0$, where the norm is of course taken with respect to the Levi form. As $M$ is strictly pseudoconvex we must have $H_{i}{ }_{j}=0$. By $[3,(5.19)]$ the entire second fundamental form of $M$ vanishes. From an analogue of the Gauss equation for the intrinsic connection we may conclude that $M$ is pseudoconformally flat (see [3, (5.13)]).

The last assertion of the theorem follows as in [9].

\section{REFERENCES}

1. S. S. Chern, Sur la possibilité de plonger un espace à connexion projective donné dans un espace projectif, Bull. Sci. Math. 61 (1937), 234-243; also in Selected Papers, Springer-Verlag, New York, 1978.

2. S. S. Chern and J. K. Moser, Real hypersurfaces in complex manifolds, Acta Math. 133 (1974), 219-271. 
3. M. J. Markowitz, CR-hypersurfaces in a space with a pseudoconformal connection, Trans. Amer. Math. Soc. 276 (1983), 117-132.

4. The local imbedding problem for conformal connections (to appear).

5. T. Ochiai, Geometry associated with semisimple flat homegeneous spaces, Trans. Amer. Math. Soc. 152 (1970), 159-193.

6. W. Rudin, Function theory in the unit ball of $\mathbf{C}^{n}$, Springer-Verlag, New York, 1980.

7. N. Tanaka, On generalized graded Lie algebras and geometric structures.I, J. Math. Soc. Japan 19 (1967), 215-254. MR 36 \#470

8. S. M. Webster, On mapping an $n$-ball into an $(n+1)$-ball in complex space, Pacific J. Math. 81 (1979), 267-272.

9.

Department of Mathematics, University of Chicago, Chicago, Illinois 60637 